\title{
The Mercury Project: A High Average Power, Gas-Cooled Laser For Inertial Fusion Energy Development
}

A. Bayramian, P. Armstrong, E. Ault, R. Beach, C. Bibeau, J. Caird, R. Campbell, B. Chai, J. Dawson, C. Ebbers, A. Erlandson, Y. Fei, B. Freitas, R. Kent, Z. Liao, T. Ladran, J. Menapace, B. Molander, S. Payne, N. Peterson, M. Randles, K. Schaffers, S. Sutton, J. Tassano, S. Telford, E. Utterback

November 9, 2006

Fusion Science and Technology 
This document was prepared as an account of work sponsored by an agency of the United States Government. Neither the United States Government nor the University of California nor any of their employees, makes any warranty, express or implied, or assumes any legal liability or responsibility for the accuracy, completeness, or usefulness of any information, apparatus, product, or process disclosed, or represents that its use would not infringe privately owned rights. Reference herein to any specific commercial product, process, or service by trade name, trademark, manufacturer, or otherwise, does not necessarily constitute or imply its endorsement, recommendation, or favoring by the United States Government or the University of California. The views and opinions of authors expressed herein do not necessarily state or reflect those of the United States Government or the University of California, and shall not be used for advertising or product endorsement purposes. 


\title{
THE MERCURY PROJECT: A HIGH AVERAGE POWER, GAS-COOLED LASER FOR INERTIAL FUSION ENERGY DEVELOPMENT
}

\author{
A. Bayramian, P. Armstrong, E. Ault, R. Beach, C. Bibeau, J. Caird, R. Campbell, B. Chai**, J. Dawson, C. Ebbers, \\ A. Erlandson, Y. Fei ${ }^{* *}$, B. Freitas, R. Kent, Z. Liao, T. Ladran, J. Menapace, B. Molander, S. Payne, N. Peterson, \\ M. Randles ${ }^{*}$ K. Schaffers, S. Sutton, J. Tassano, S. Telford, and E. Utterback \\ Lawrence Livermore National Laboratory, 7000 East Ave., L-470, Livermore, CA 94551, email: bayramian1@llnl.gov \\ "Northrop Grumman Space Technologies, Synoptics, 1201 Continental Blvd., Charlotte, NC 28273 \\ ${ }^{* *}$ Crystal Photonics, Inc 5525 Sanford Lane, Sanford, Fl 32773
}

Hundred-joule, kilowatt-class lasers based on diodepumped solid-state technologies, are being developed worldwide for laser-plasma interactions and as prototypes for fusion energy drivers. The goal of the Mercury Laser Project is to develop key technologies within an architectural framework that demonstrates basic building blocks for scaling to larger multi-kilojoule systems for inertial fusion energy (IFE) applications. Mercury has requirements that include: scalability to IFE beamlines, $10 \mathrm{~Hz}$ repetition rate, high efficiency, and $10^{9}$ shot reliability. The Mercury laser has operated continuously for several hours at $55 \mathrm{~J}$ and $10 \mathrm{~Hz}$ with fourteen $4 \times 6 \mathrm{~cm}^{2}$ ytterbium doped strontium fluoroapatite (Yb:S-FAP) amplifier slabs pumped by eight 100 $\mathrm{kW}$ diode arrays. The $1047 \mathrm{~nm}$ fundamental wavelength was converted to $523 \mathrm{~nm}$ at $160 \mathrm{~W}$ average power with 73 $\%$ conversion efficiency using yttrium calcium oxy-borate (YCOB).

\section{INTRODUCTION}

The National Ignition Facility (NIF), currently under construction and activation at Lawrence Livermore National Lab (LLNL), provides unprecedented levels of laser energy for stockpile stewardship applications, research in high energy density physics, and is widely anticipated to demonstrate inertial confinement fusion (ICF) ignition with net energy gain. Hundred-joule, kilowatt-class lasers based on diode-pumped solid-state technologies are being developed worldwide for research in laser-plasma interactions as well as for IFE prototype drivers. The Mercury laser project goals are to develop the required key technologies at sub-scale energies and beamline apertures, within an architectural framework that represents the building blocks of larger multikilojoule systems. These designs have requirements that include: scalability to IFE beamlines, $10 \mathrm{~Hz}$ repetition rate, high efficiency and $10^{9}$ shot reliability. Orth, Payne and Krupke proposed these design criteria in a paper where the cost of electricity was derived from component level efficiencies and costs. ${ }^{1}$ Target designs are continually being optimized with higher gains and/or lower drive energies, but the essential requirements on the laser driver remain the same. As the Mercury system nears completion, the technologies used to modify the output spatially, spectrally, and temporally are being implemented. We are currently building a user facility in a separate lab, where diverse target experiments can be performed. One of the first planned experiments will generate $\mathrm{x}$-rays at $10 \mathrm{~Hz}$ that can be used to probe dynamic phase transitions in shocked materials.

\section{ARCHITECTURE}

The transition from a flashlamp pumping to diode pumping is fundamental to the cost, reliability, and efficiency of a megajoule class solid-state laser.. The Mercury pump architecture uses an end-pumped scheme that takes advantage of high brightness diode arrays. The diode light from large $100 \mathrm{~kW}$ arrays $\left(\sim 1 \mathrm{~kW} / \mathrm{cm}^{2}\right)$ is first angularly redistributed by hollow concentrating optics to a $3 \times 5 \mathrm{~cm}$ aperture $\left(10 \mathrm{~kW} / \mathrm{cm}^{2}\right)$ with homogeneous angular divergence in both the $\mathrm{x}$ and $\mathrm{y}$ directions. The diode light is then guided by a rectangular reflective cavity called a 'homogenizer', which maintains the angular distribution while spatially smoothing the diode pump profile. Since diodes are brightness limited sources, efficient use of the pump radiation requires a gain medium with a long radiative lifetime, which was one of the primary selection criteria for the gain medium of this advanced average power IFE driver. The homogeneous pump radiation then excites a compact amplifier cassette containing several thin slabs of gain media that are cooled by high speed helium gas, thus enabling the required 10 $\mathrm{Hz}$ repetition rate. The gas cooling creates a longitudinal thermal gradient in the amplifier slabs such that to first order there is no radial gradient in the thermal profile and therefore minimal induced thermal wavefront distortion as 
well. The gain is then extracted by the $1047 \mathrm{~nm}$ front end laser which propagates longitudinally down the center of the duct, homogenizer, where it is then amplified in the amplifier slab cassette (Fig. 1). In total, the Mercury laser consists of two of these amplifier assemblies each of which is pumped by four $100 \mathrm{~kW}$ diode arrays to produce 12.3 nepers $\left(2.2 \times 10^{5}\right)$ of total gain with $100 \mathrm{~J}$ of potential output energy. To extract the $100 \mathrm{~J}$ with a modest frontend input of $100 \mathrm{~mJ}$ requires four passes through the dual amplifier configuration. The four pass architecture is enabled by the passive method of angular multiplexing or off-axis relay imaging to overlay images at the amplifier locations while maintaining the ability to inject and pickoff the beams near focus where the angle multiplexing causes beam separation. Each pass uses custom pinholes tailored in size for optimal spatial filtering while accommodating high power. Relay imaging is used to increase reliability of the amplifiers by minimizing intensity modulations that would increase the probability of optical damage. Although four passes can be achieved in this architecture without the need for an optical switch, there is still a need to prevent parasitics due to the large total gain. To control these parasitics, a Pockels cell is placed in the system after two passes which requires the cell be able to handle $200 \mathrm{~W}$ average power while maintaining $>200: 1$ contrast at a fluence of $2 \mathrm{~J} / \mathrm{cm}^{2}$. A thermal birefringence compensated Pockels cell that meets these requirements was developed and commissioned on the Mercury laser. ${ }^{2}$ Due to the $10 \mathrm{~Hz}$ repetition rate and relatively high fluences possible in the amplifiers, a suite of high speed, high resolution diagnostics allows interrogation of the beam on each pass in order to monitor for optical damage or anomalous behavior. In particular, CCD cameras and real-time image differencing algorithms allow rapid detection of beam anomalies at $10 \mathrm{~Hz}$ and shut down the system before the next shot.

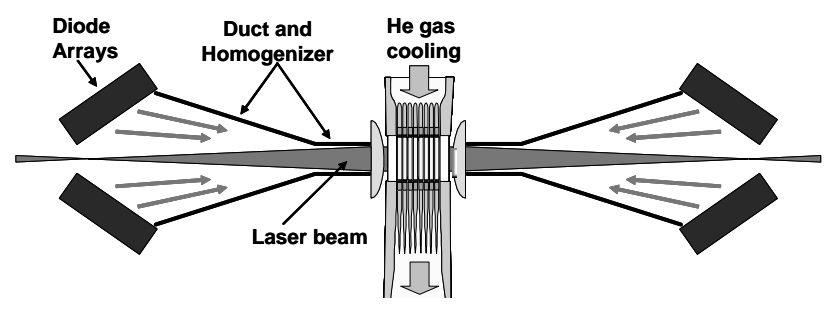

Fig. 1 Diode pump, cooling, extraction architecture for Mercury amplifiers

\section{AMPLIFIER TECHNOLOGIES: DIODES, CRYSTALS, AND GAS-COOLING}

Each of these three technologies has matured to the point where the basic needs of the laser are satisfied and the focus has shifted to optimization and scaling for the next generation kilojoule class system. The diode arrays heavily leverage mass production and commercial markets for diodes. With the commercialization of the LLNL diode packaging ${ }^{3}$ by Coherent Inc, an entire high brightness array could be procured. The arrays already being used in the laser meet all of the basic requirements (see table 1) except wallplug efficiency (45\% versus 50 $\%)$. However, this is not a problem for future systems, since recent tests of commercial bars have shown wallplug efficiencies of $65 \%$ at 200 W/bar with reliability exceeding current requirements.

Table I Diode array requirements

\begin{tabular}{|l|c|c|}
\hline Attribute & Requirement & Performance \\
\hline Power & $>100 \mathrm{~W} / \mathrm{bar}$ & $120 \mathrm{~W} / \mathrm{bar}$ \\
\hline Reliability (shots) & $>2 \times 10^{8}$ & $1.4 \times 10^{8}$ \\
\hline Power droop (1 msec) & $<15 \%$ & $4.3 \%$ \\
\hline Linewidth & $<5 \mathrm{~nm}$ & $2.3 \mathrm{~nm}$ \\
\hline Linewidth (1 msec) & $<8.5 \mathrm{~nm}$ & $4.1 \mathrm{~nm}$ \\
\hline Wallplug efficiency & $>50 \%$ & $45 \%$ \\
\hline
\end{tabular}

Yb:S-FAP $\left(\mathrm{Yb}^{3+}: \mathrm{Sr}_{5}\left(\mathrm{PO}_{4}\right)_{3} \mathrm{~F}\right)$ crystals have many attractive laser properties that make them well suited for diode pumping in moderate thermal load applications ${ }^{4}$. Mercury is now operational with 14 slabs in the system, each with dimensions $4 \mathrm{~cm}$ x $6 \mathrm{~cm}$ x $0.75 \mathrm{~cm}$. Significant progress has been made toward reducing or eliminating the two remaining defects (grain boundaries and bubble core) to produce high quality material. Detailed thermal models of the furnace design and growth conditions are being developed to allow understanding and correction of defect formation as well as transitioning current boule sizes to larger scales needed for inertial fusion energy applications. In addition, a comprehensive laser induced defect initiation and characterization activity has been started to monitor improvements in crystal quality and provide additional feedback for improvement of the growth and modeling efforts. The primary reason for laser induced defect characterization is to establish probability functions for the long-term $\left(10^{9}\right.$ shot $)$ survivability of the amplifiers and system optics. These statistics are needed for predicting the overall reliability and availability of an IFE power plant as well as cost of optical components for the life of the plant. The first large diameter scaling of Yb:S-FAP was accomplished this year with the first 12 $\mathrm{cm}$ diameter Czochralski grown boules. The largest SFAP slab ever fabricated was harvested from the boule producing a $6 \times 8 \times 1.4 \mathrm{~cm}^{3}$ slab (Fig. 2a).

The technology that enables $10 \mathrm{~Hz}$ operation of the laser is the sub-sonic helium gas flow. The amplifier cassettes are composed of seven aerodynamic "vanes" that contain the S-FAP slabs. The vanes are assembled together with each vane separated by a one-millimeter wide helium gas-cooling channel to form the amplifier cassette. At 50 psi and 0.1 Mach flow velocity, the 
cooling system removes $1-3 \mathrm{~W} / \mathrm{cm}^{2}$ from the faces of each slab. The thermally induced wavefront distortion at $900 \mu$ s pumping (> $100 \%$ of design point operation), has been measured experimentally to be $\sim 1$ wave peak to valley in each amplifier or $\sim 8$ waves for the total system. By adding the windows and lenses to the thermal model of the amplifier, the theory predicts values that closely match the experiment in both magnitude and spatial profile (Fig. 2b). This wavefront distortion can be corrected by either static or active correction providing the equivalent of single shot beam quality at up to $1 \mathrm{~kW}$ of average power. The advantage of this cooling method is that residual thermally induced distortion is a consequence of the edges of the amplifier, thus scaling to larger apertures will further reduce this distortion. a)

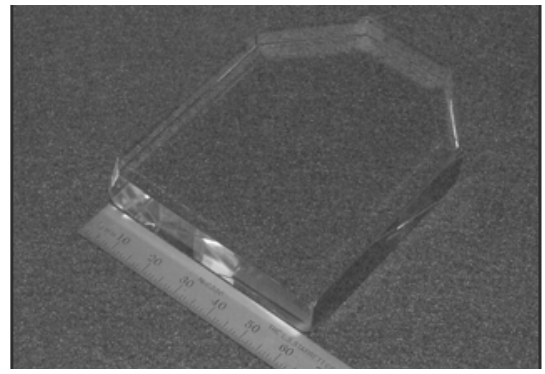

Theory

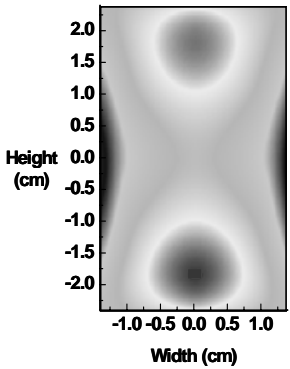

b)

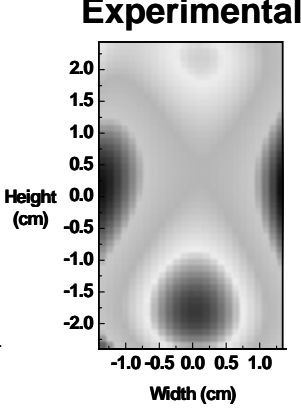

$1.0 \lambda$

0.5

0
Fig. 2. a) $6 \times 8 \times 1.4 \mathrm{~cm}^{3}$ slab harvested from a $12 \mathrm{~cm}$ diameter boule, b) Theoretical and experimental thermal wavefront distortion in an amplifier head at full pumping conditions showing $\sim 1$ wave of distortion.

\section{LASER OPERATION}

The most recent set of experiments focused on demonstrating performance at $0.6 \mathrm{~kW}$. To commission the laser, the gain was first increased changing the diode pulse length from 300 to $900 \mu$ sec (Fig. 3a). At each operating point the system was tested for parasitics and ASE losses. The collection of data allowed a mapping of the system gain that compared favorably with energetics modeling. The front-end system provides temporal pulse shaping capability to compensate for the square pulse distortion caused by gain saturation giving a nearly square pulse in time at the output (Fig. 3a). At a maximum gain of approximately 22 per pass, the front-end injection energy was gradually increased. The average power of the system was ramped as well with single shot checks followed by $1 \mathrm{~Hz}, 5 \mathrm{~Hz}$, and finally $10 \mathrm{~Hz}$ operations for up to several hours. Over several campaigns the system operated at the $55 \mathrm{~J}$ output level at $10 \mathrm{~Hz}$ for a cumulative total of seven hours and in a recent campaign at over $61 \mathrm{~J}$ for several minutes (Fig. 3b). In all cases a controlled shutdown was employed with no evidence of optical damage in the system.

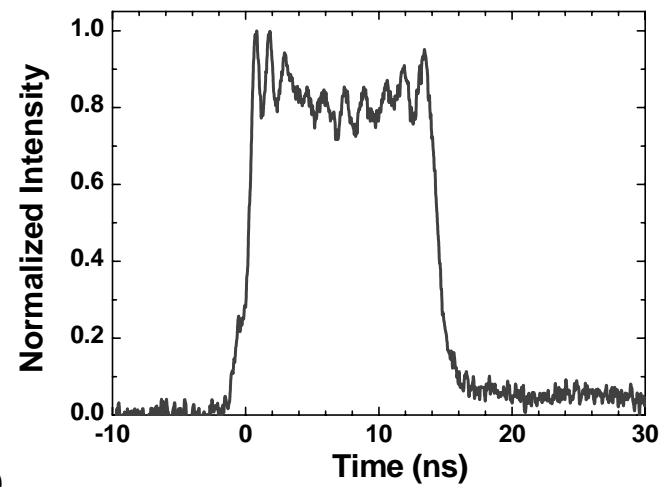

a)

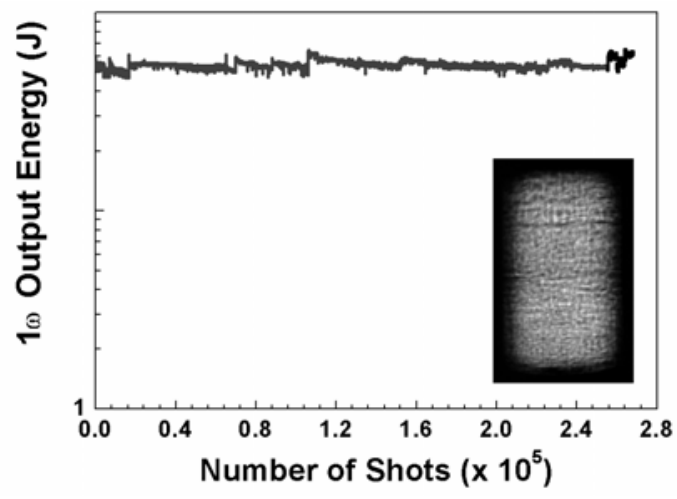

Fig. 3 a) 14 ns FWHM shaped output laser pulse, b) Average power operation at both $55 \mathrm{~J}$ and $61 \mathrm{~J}$ with a cumulative total of $2.7 \times 10^{5}$ shots

\section{ADVANCED TECHNOLOGIES: AVERAGE POWER FREQUENCY CONVERSION, FRONT END, AND DEFORMABLE MIRROR}

Typical absorption for transparent nonlinear crystals is on the order of $10^{-3} / \mathrm{cm}$, leading to an average power loading of $0.01 \mathrm{~W} / \mathrm{cm}^{2}$ in Mercury. The modest thermal load leads to relatively small temperature gradients of $2-3^{\circ} \mathrm{C}$ in the nonlinear crystal. Nevertheless, the gradient can lead to thermal dephasing (reduced conversion efficiency) or thermal fracture in the case of more fragile nonlinear materials such as KDP. At these low thermal loads, the aggressive cooling method used on the amplifiers is not needed. Instead we use a method that relies on a thermally conductive and transparent substrate ${ }^{5}$. We have chosen sapphire since it is widely used as an optical window 
substrate and has a relatively high thermal conductivity $(30 \mathrm{~W} / \mathrm{m} / \mathrm{K})$. The scalable frequency conversion cooler concept (Fig. 4a) uses close coupling of the crystal to the sapphire plate. However, at the current aperture of the laser, the cooling can be simplified further with YCOB due to its low absorption by simply edge cooling the slab. Using edge cooling at $500 \mathrm{~W}$ average power $(1047 \mathrm{~nm})$ loading we see no degradation of the output power at 523 $\mathrm{nm}$ as a function of time indicating the thermal loading and resultant distortion in the crystal is within the temperature acceptance bandwidth of YCOB.

a)
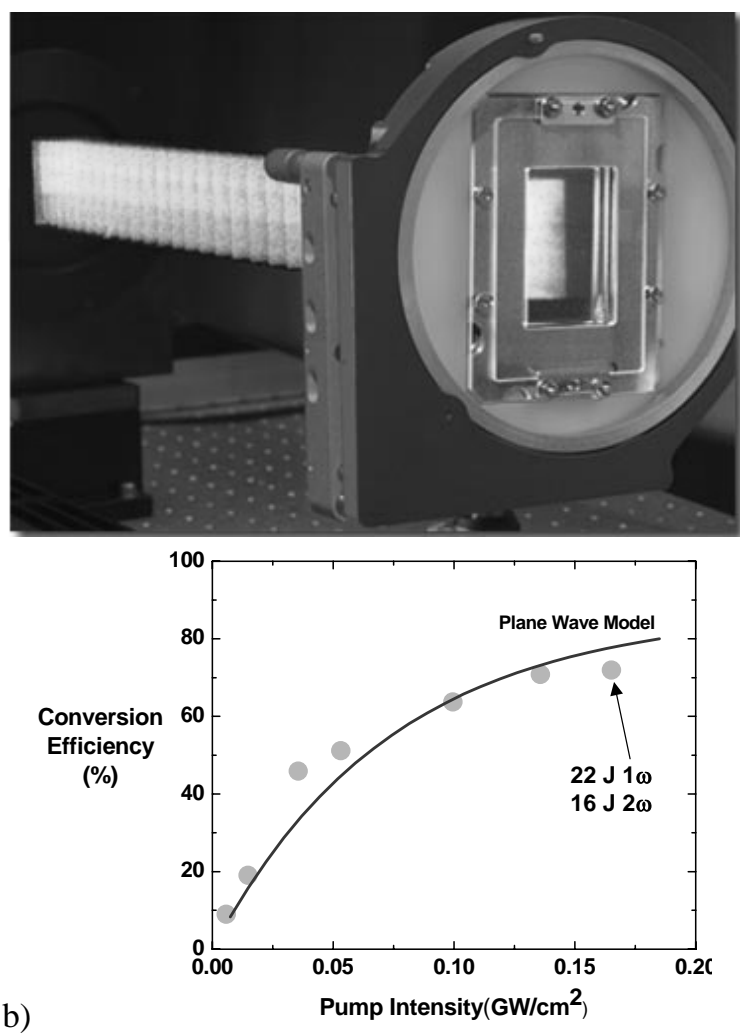

Fig. 4. a) Average power frequency conversion module showing conversion to $523 \mathrm{~nm}$, b) Conversion efficiency versus drive intensity showing maximum conversion efficiency of $73 \%$ with plane wave model overlay for comparison

The growth of the YCOB material has also progressed with commercial production of the $5.5 \times 8.5$ $\mathrm{cm}^{2}$ aperture required for the Mercury laser. In addition to its enhanced thermo-mechanical properties, YCOB also has 3 times the $\mathrm{d}_{\text {eff }}$ relative to $\mathrm{KDP}^{6,7}$, which decreases the required material thickness or drive intensity requirements and increases angular acceptance of a high average power frequency converter based on YCOB. In the first full power frequency conversion experiment, the Mercury output beam was image relayed to a side-cooled AR coated YCOB crystal $1.58 \mathrm{~cm}$ thick (Fig. 4a). We demonstrated record conversion efficiencies for the Mercury laser system of $73 \%$ at an incident power of 220 $\mathrm{W}$ at $1047 \mathrm{~nm}$ producing $160 \mathrm{~W}$ at $523 \mathrm{~nm}$ (Fig. 4b). A theoretical prediction based on a plane wave model is used to illustrate that the Mercury laser beam quality and intensity profile are both uniform enough and within the temperature and angular acceptance of the YCOB converter to yield nearly the maximum theoretical frequency conversion efficiency. In the next few months, the $1 \omega$ drive will be increased to $60 \mathrm{~J} @ 10 \mathrm{~Hz}$ to produce $420 \mathrm{~W}$ of $2 \omega$ light.

The front-end laser system being developed for Mercury will produce $500 \mathrm{~mJ}$ at $10 \mathrm{~Hz}$ with flexibility in temporal, spectral, and spatial sculpting. The output pulse width can be varied from 2 to 15 nanoseconds with arbitrary temporal pulse-shaping capability which can be used to compensate for square pulse distortion or provide user define pulseshapes at the output of Mercury. Spectral bandwidth is needed for injection into Mercury to allow beam smoothing of a far field spot, decrease coherent effects, and prevent the buildup of nonlinear parasitics such as Stimulated Raman Scattering (SRS) and Stimulated Brillouin Scattering (SBS). RF bandwidth up to $250 \mathrm{GHz}$ is applied to the beam at low energy by double passing the beam through a lithium niobate phase modulator. The modulation side bands are individually attenuated to compensate for the gain profile in SFAP, thereby minimizing amplitude modulation that would normally result from the gain-narrowing of a frequency modulated spectrum ${ }^{8}$. The stability and energy are achieved in this front end through a combination of fiber amplifiers and a relay imaged multipass power amplifier. The front-end system starts with a fiber oscillator and three large mode area ytterbium doped fiber amplifiers to produce up to $30 \mu \mathrm{J}$. This output is then injected into a relay imaged optical ring cavity with two Yb:S-FAP amplifiers. The complete system produces more than 525 mJ output (Fig. 5a) with $150 \mathrm{GHz}$ RF bandwidth with $80 \%$ of energy in a 2 times diffraction limited spot.

The high average power operation of the Mercury laser induces dynamic thermal aberrations to the output beam wavefront and far field as indicated in section III Fig. 2. To compensate for the potential 2-3 waves per pass of low order aberration and regain an optimal far field spot, an active corrective optic is needed. Using a 4 wave lateral shearing wavefront sensor from Phasics Inc. and a $10 \mathrm{~cm}$ diameter bimorph deformable mirror (41 actuators) from Night N Opt., we can actively correct for dynamic distortions due to the thermal loading as well as correct for static distortions of the entire system. The mirror/sensor combination has been commissioned on the Mercury laser at over $600 \mathrm{~W}$ output power (fig. 5b). With optimized correction, the far field spot size was reduced from $>7$ times diffraction limited to $<4.5$ times diffraction limited which meets our design requirements. 


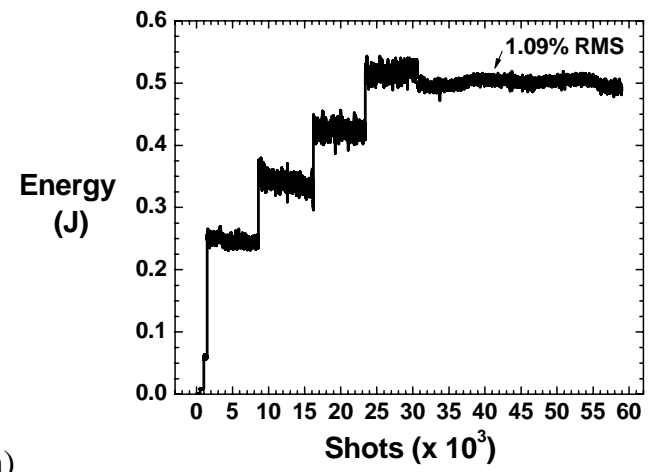

a)

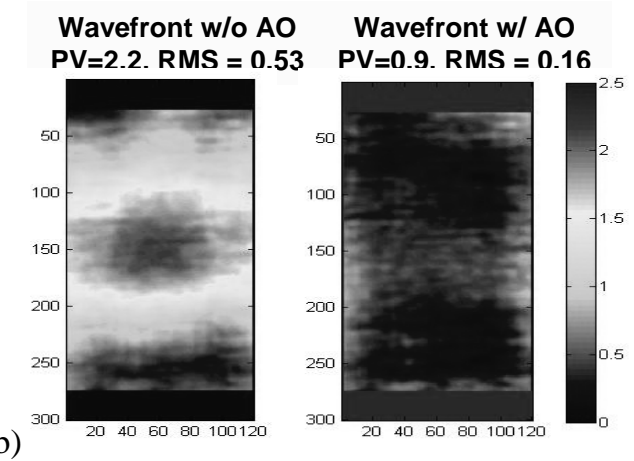

Fig. 5 a) Front end output energy showing $525 \mathrm{~mJ}$ maximum output energy with a stability of $1.09 \% \mathrm{rms}$, b) Output wavefront of the laser at $300 \mathrm{~W}$ output power without and with correction by the adaptive optic.

\section{CONCLUSION}

A critical goal of the Mercury laser project is to demonstrate the technologies necessary for scaling up to multi-kilojoule apertures relevant for IFE. The next steps require increasing the diode array size and laser aperture to a full IFE beamline, diode and system cost reduction, long term reliability, demonstrating a scaled full power Pockels cell, and incorporating beam bundling. We are constructing a Mercury based user facility for conducting both IFE related experiments as well as basic research pursuits in the areas of high energy density physics, laser plasma interactions, and both photon and particle effects on IFE optics and chamber materials. The $10 \mathrm{~Hz}$ repetition rate of this laser system will allow parametric studies, the effect of long-term radiation on materials, and averaging to enhance signal to noise. The commissioning of such a facility necessitates development efforts in target manipulation, target tracking, and diagnostics capable of handling the $10 \mathrm{~Hz}$ repetition rate that are also required technologies for IFE. The first experiment will utilize the Mercury laser beam for optical lifetime testing of materials and surfaces for the IFE final optic. When the facility is completed, initial focused target experiments will include $10 \mathrm{~Hz} \mathrm{X}$-ray radiography and structural characterization of shocked material.

\section{ACKNOWLEDGEMENTS}

This work was performed under the auspices of the U.S. Department of Energy by the University of California Lawrence Livermore National Laboratory under contract No. W-7405-Eng-48.

\section{REFERENCES}

1. C. D. Orth, S. A. Payne, and W. F. Krupke, "A diode pumped solid state laser driver for inertial fusion energy," Nuc. Fus. 36, 75 (1996).

2. C.A. Ebbers, K. Kanz, and H. Nakano," A thermally compensated, deuterated KDP Q-switch for high average power lasers," Conference for Lasers and Electro Optics, Baltimore Maryland, (2002).

3. J.A. Skidmore, et. al, "Silicon monolithic microchannel-cooled laser diode array," Appl. Phys. Lett., 77, 10 (2000).

4. K.I. Schaffers, et al., "Growth of Yb:S-FAP [Yb3+:Sr5(Po4)3F] crystals for the Mercury laser,” $J$. Cryst. Growth, 253, 297 (2003).

5. Randall J., et al., "Diode-array-pumped kilowatt laser,” SPIE Proc. 3264, 2 (1998).

6. Iwai M, et al., "Growth and characterization of GdCOB and YCOB as new nonlinear optical materials,” Jap. JAP. 36, 276 (1997).

7. 6. G. Aka et al. "A new nonlinear and neodymium laser self-frequency doubling crystal with congruent melting Ca4GdO(BO3)3, (GdCOB)", Eur. J. Solid State Inorg. Chem., 33, 727 (1996)

8. L.J. Waxer, et al., "Precision spectral sculpting for narrow-band amplification of broadband frequencymodulated pulses, Opt. Lett., 27, 1427 (2002). 